Bundesgesundheitsbl 2018 · 61:1012-1021 https://doi.org/10.1007/s00103-018-2784-1 Online publiziert: 16 . Juli 2018

(c) Der/die Autor(en) 2018

CrossMark

\author{
Maria Flothkötter ${ }^{1}$ Julia Kunath ${ }^{1} \cdot$ Stephanie Lücke ${ }^{1}$ Katharina Reiss ${ }^{1} \cdot$ \\ Juliane Menzel ${ }^{2} \cdot$ Cornelia Weikert $^{2}$ \\ 'Netzwerk Gesund ins Leben/Bundeszentrum für Ernährung (BZfE) in der Bundesanstalt für \\ Landwirtschaft und Ernährung (BLE), Bonn, Deutschland \\ ${ }^{2}$ Bundesinstitut für Risikobewertung, Berlin, Deutschland
}

\title{
Das internationale Forschungsvorhaben Becoming Breastfeeding Friendly. Untersuchung von Rahmenbedingungen zur Stillförderung
}

\section{Hintergrund des Forschungs- vorhabens}

Die ersten 1000 Tage im Leben eines Kindes - die Zeit von der Konzeption bis zum Kleinkindalter von etwa zwei Jahren - bieten besondere Chancen, frühzeitig die Weichen für eine langfristige Gesundheit zu stellen. Nationale und internationale Expertengruppen empfehlen, das Stillen als natürliche und bevorzugte Ernährungsform für Säuglinge zu fördern, wobei jegliches - auch teilweises - Stillen sinnvoll ist [1]. Muttermilch ist ideal an die kindlichen Bedürfnisse angepasst - sie hat die optimale Zusammensetzung, ist hygienisch einwandfrei, richtig temperiert, immer verfügbar und kostet nichts. Viele Studien und systematische Arbeiten zeigen, dass Stillen mit kurz- und langfristigen gesundheitlichen Vorteilen für Kind und Mutter assoziiert ist [2-6]. In einer Veröffentlichung von Victora und Kollegen (2016) wurden die positiven Auswirkungen des Stillens basierend auf 28 systematischen Reviews und Metaanalysen verdeutlicht [7]. Die Analysen zeigten eine protektive Wirkung bei Infektionen und Abweichungen von der normalen Zahnstellung (Malokklusion) bei gestillten Kindern, eine gesteigerte Intelligenz sowie eine mögliche Vermeidung von späterer Adipo- sitas und Diabetes gegenüber Kindern, die nicht oder nur für einen kurzen Zeitraum gestillt wurden [7]. Des Weiteren konnte die Forschergruppe erneut belegen, dass stillende Frauen seltener an Brustkrebs, Eierstockkrebs und Diabetes Typ 2 erkranken [7]. Trotz dieser Vorteile werden in Ländern mit mittlerem sowie hohem Einkommen im Durchschnitt lediglich $37 \%$ der Kinder entsprechend dem empfohlenen Zeitraum gestillt [7, 8]. Diese Ergebnisse decken sich mit Daten aus Deutschland. Nach den Daten des Kinder- und Jugendgesundheitssurveys (KiGGS Welle 1) des Robert KochInstituts (RKI) beginnen zwei Drittel aller Mütter nach der Geburt eines Kindes in Deutschland ausschließlich zu stillen. Nach vier Monaten werden nur noch $38 \%$ der Kinder ausschließlich mit Muttermilch ernährt, nach sechs Monaten $12 \%$ [9]. Die sehr geringen Prävalenzen für vier- und sechsmonatiges ausschließliches Stillen zeigen die Notwendigkeit von nachhaltiger Unterstützung stillender Mütter über die Zeit kurz nach der Geburt hinaus [10, 11].

Eine bedeutende Rolle in der Stillförderung spielen unter anderem die Innocenti-Deklaration von 1990, die von 30 Staaten und verschiedenen internationalen Organisationen verabschiedet wurde, „zum Schutz, zur Förderung und
Unterstützung des Stillens" [12] sowie die „Baby-friendly Hospital Initiative“ (BFHI) der Weltgesundheitsorganisation (WHO) und des Kinderhilfswerks der Vereinten Nationen (UNICEF; [13]). Aufbauend auf den Empfehlungen der „Globalen Strategie zur Ernährung von Säuglingen und Kleinkindern" der WHO wurde in einem Projekt der Europäischen Union (EU) ein Aktionsplan zum Schutz, zur Förderung und Unterstützung des Stillens in Europa entwickelt, der als Modell für nationale Aktionspläne dienen sollte. Darüber hinaus ist die weltweite Stillförderung unter anderem ein globales Ernährungsziel der UN-Dekade "Action on Nutrition“, die die World Health Assembly (WHA) für den Zeitraum 2016 bis 2025 ausgerufen hat [14]. Demnach soll die Rate ausschließlichen Stillens in den ersten sechs Lebensmonaten bis 2025 auf mindestens $50 \%$ steigen [14]. Trotz verschiedener Initiativen bleiben die weltweiten Stillraten weit unter den internationalen Zielen [8, 15]. Ob und wie viele Mütter stillen, ist von verschiedenen Faktoren abhängig. So tragen Probleme nach der Geburt, ein Übergewicht der Mutter vor bzw. zu Beginn der Schwangerschaft, das Alter, die Vermarktung von Muttermilchersatzprodukten oder das Rauchen der Mutter während der Schwangerschaft dazu bei, 


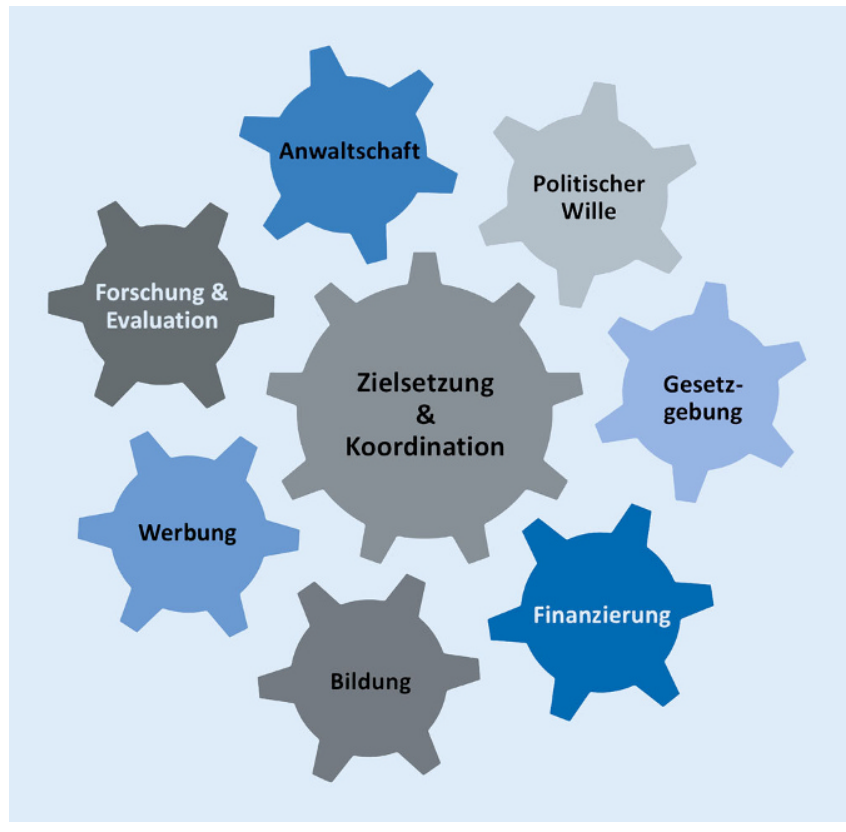

Abb. $1<$ Handlungsfelder der Stillförderung nach dem Breastfeeding Gear Model. Abbildung modifiziert nach [25], @ 2012 American Society for Nutrition

dass Kinder seltener gestillt werden [10, 11, 16]. Die Gründe zum Abstillen reichen von ganz persönlichen Motiven bis hin zu einem Gefühl, dass Stillen öffentlich nicht besonders erwünscht ist. Demnach ist die Förderung eines stillfreundlichen Klimas in der Gesellschaft unbedingt notwendig [17]. Aber auch der sozioökonomische Status (Einkommen, Bildung und Beschäftigung) der Mutter hat einen entscheidenden Einfluss $[10,11]$. So stillen Mütter mit einem hohen sozioökonomischen Status deutlich häufiger und länger als Mütter mit einem niedrigen sozioökonomischen Status [11, 18]. Trotz vereinzelter Stillfördermaßnahmen in Deutschland wird gerade diese vulnerable Gruppe nur schlecht erreicht.

Verschiedene Initiativen und Programme verfolgen das Ziel der Stillförderung. Die Global Breastfeeding Scorecard von WHO und UNICEF dokumentiert Schlüsselindikatoren der Politik und von Programmen, die sich auf den Schutz, die Unterstützung und die Förderung des Stillens in einem Land auswirken, und informiert über die aktuellen Stillraten weltweit. Sie soll den Fortschritt fördern, die Rechenschaftspflicht erhöhen und den Wandel für alle Länder dokumentieren, die die notwendigen Maßnahmen zum Schutz, zur Förderung und zur Unterstützung des Stillens ergreifen [19]. Die vom In- ternational Baby Food Action Network (IBFAN) ins Leben gerufene „World Breastfeeding Trends Initiative" (WBT $i$ ) ist ein Instrument, das zehn Indikatoren der Politik und von Programmen verfolgt, die Frauen dabei unterstützen, erfolgreich $\mathrm{zu}$ stillen. WBTi regt alle drei bis fünf Jahre zu einer Neubewertung an, um Trends $\mathrm{zu}$ untersuchen. Hierbei identifiziert eine Kerngruppe in Zusammenarbeit mit lokalen Partnern bestehende Lücken in der Politik und regt zu Maßnahmen an. WBTi hilft so, eine praktische Grundlage zu schaffen, die den Projektplanern und politischen Entscheidungsträgern zeigt, wo Verbesserungen erforderlich sind, um die Ziele der globalen Strategie der WHO zu erreichen. Die Ergebnisse der einzelnen Länder werden auf der WBTi-Website veröffentlicht, was den Regierungschefs weitere Impulse zu entschlossenem Handeln gibt [20].

Das im September 2017 in Deutschland gestartete Forschungsvorhaben $\mathrm{Be}$ coming Breastfeeding Friendly (BBF), das in diesem Beitrag vorgestellt wird, entstand auf Initiative des Bundesministeriums für Ernährung und Landwirtschaft (BMEL) und wird vom Netzwerk Gesund ins Leben im Bundeszentrum für Ernährung (BZfE) und der Nationalen Stillkommission am Bundesinstitut für Risikobewertung (BfR) gemeinsam mit der Yale School of Public Health durchge- führt. Im Vergleich zu den bestehenden standardisierten Erhebungsinstrumenten wie der Global Breastfeeding Scorecard und der WBTi $[14,21,22]$ geht das Projekt BBF über ein deskriptives Monitoring des aktuellen Stands hinaus. Denn Stakeholder aus unterschiedlichen Handlungsfeldern der Stillförderung formulieren darauf aufbauend gemeinsame Handlungsempfehlungen und konkrete Vorschläge zur Implementierung im eigenen Land [23]. Bereits der Diskussionsprozess setzt positive Impulse frei. Das zugrunde liegende Modell beschreibt, wie acht spezifische Handlungsfelder (symbolisiert durch Zahnräder) ineinandergreifen und möglichst harmonieren müssen, um den Erfolg von Stillförderprogrammen zu maximieren (• Abb. 1; [25]). Jedes dieser acht Handlungsfelder wird innerhalb des Prozesses nach einer standardisierten Methode evaluiert, wodurch ersichtlich wird, in welchem Bereich Unterstützung gebraucht wird. [23]. Die einflussreichsten Indikatoren einer erfolgreichen Stillförderung wurden in einem wissenschaftlichen Konsensverfahren von Experten identifiziert [23].

\section{Ziel des Vorhabens}

Durch das internationale Forschungsvorhaben BBF sollen die Rahmenbedingungen für das Stillen in Deutschland verbessert werden. Insbesondere in föderalistisch geprägten Ländern wie Deutschland existiert eine oft unübersichtliche Vielzahl von Projekten, Initiativen und Strategien zu Themen mit Gesundheitsbezug, einschließlich des Themas Stillen. Es fehlt jedoch eine umfassende und systematische Bestandsaufnahme aller Strukturen, Akteure und Maßnahmen zur Stillförderung in Deutschland. An genau dieser Stelle setzt das Projekt BBF an. In einem zweijährigen Prozess wird systematisch durch eine Stärken-SchwächeAnalyse ermittelt, wo genau eine wirksame, effiziente und nachhaltige Stillförderung gezielt ansetzen sollte. Das Vorhaben ist auf insgesamt 24 Monate ausgelegt. In dem vorliegenden Artikel werden das Projekt und die ihm zugrunde liegende Konzeption vorgestellt. 
Das Forschungsvorhaben widmet sich den folgenden Teilzielen:

1. Vollständige Abbildung der Istsituation der Stillförderung in Deutschland

2. Bewertung der Istsituation im Hinblick auf Stärken und Schwächen inklusive Ableitung von Handlungsbedarfen und -empfehlungen

3. Planung und Implementierung konkreter Maßnahmen zur Stillförderung aufbauend auf Schritt 1 und 2

4. Öffentlichkeitswirksame Kommunikation zur Verbreitung der Informationen über den Nutzen des Stillens und zur Verbesserung der Akzeptanz (Image) des Stillens

\section{Methoden}

Das Forschungsvorhaben Becoming Breastfeeding Friendly ermöglicht eine länderspezifische Beurteilung über die Einflussfaktoren des Stillens durch evidenzbasierte Kriterien und stellt eine Anleitung zur Erfassung der Handlungsbedarfe im Bereich der Stillförderung zur Verfügung. Praktische Hilfestellungen und Prozessanleitungen sowie Anwendungsbeispiele zum Programm sind weltweit einsehbar und können von jedem Land angepasst und angewendet werden. Anders als bisher existierende Initiativen, liefert das Projekt evidenzbasierte Informationen, die direkt für politische Entscheidungsprozesse zur Stillförderung genutzt und umgesetzt werden können [24].

\section{Das Zahnradmodell}

Die theoretische Grundlage des Forschungsvorhabens ist das von Prof. Rafael Pérez-Escamilla und seinem Team an der Yale School of Public Health entwickelte Breastfeeding Gear Model (BFGM; - Abb. 1), welches auf einer systematischen Literaturrecherche und -auswertung wissenschaftlicher, einschließlich grauer Literatur basiert [23, 25]. Das Modell besteht aus acht Zahnrädern (Gears), die die unterschiedlichen Handlungsfelder der Stillförderung darstellen:

- Anwaltschaft,

- politischer Wille,

- Gesetzgebung,

Bundesgesundheitsbl 2018 61:1012-1021 https://doi.org/10.1007/s00103-018-2784-1

(c) Der/die Autor(en) 2018

M. Flothkötter · J. Kunath · S. Lücke · K. Reiss · J. Menzel · C. Weikert

\section{Das internationale Forschungsvorhaben Becoming Breastfeeding Friendly. Untersuchung von Rahmenbedingungen zur Stillförderung}

\section{Zusammenfassung}

Trotz bekannter positiver Auswirkungen für gestillte Kinder und ihre Mütter wird nur etwa jeder dritte Säugling in Deutschland im Alter von vier Monaten ausschließlich gestillt. Es besteht zudem kaum ein Überblick über Strukturen, Akteure und Maßnahmen zur Stillförderung in Deutschland. In einem zweijährigen internationalen Forschungsprojekt mit dem Titel „Becoming Breastfeeding Friendly" (BBF) werden stillförderliche und -hinderliche Faktoren auf Grundlage des von der Yale School of Public Health entwickelten Breastfeeding Gear Model (BFGM) systematisch erfasst. Dazu bewerten Experten aus den Fachgesellschaften der relevanten Gesundheitsberufe, Wissenschaft, Politik und Kommunikation 54 Prüfkriterien (Benchmarks) in den acht Handlungsfeldern: Anwaltschaft, politischer Wille, Gesetzgebung, Finanzierung, Bildung, Werbung, Forschung \& Evaluation, Zielsetzung \& Koordination. Das Ergebnis ist Grundlage, um gezielt effiziente und nachhaltige Maßnahmen zur
Stillförderung zu planen und zu begleiten. Durch die Identifizierung von Stärken und Handlungsbedarfen können konkrete Handlungsempfehlungen ("calls to action") zur Stillförderung abgeleitet und priorisiert werden. Das im September 2017 gestartete Forschungsprojekt wird auf Initiative des Bundesministeriums für Ernährung und Landwirtschaft (BMEL) vom Netzwerk Gesund ins Leben und der Nationalen Stillkommission am Bundesinstitut für Risikobewertung (BfR) gemeinsam mit der Yale School of Public Health durchgeführt. Es untersucht die Rahmenbedingungen für das Stillen und liefert damit einen wertvollen Beitrag zur Gesundheitsförderung von Mutter und Kind und zum Stillschutz in Deutschland.

\section{Schlüsselwörter}

Stillförderung · Becoming Breastfeeding Friendly · Netzwerk Gesund ins Leben . Nationale Stillkommission

\section{Becoming breastfeeding friendly in Germany_an international research project to assess the readiness to scale up breastfeeding}

\section{Abstract}

Despite known positive effects for breastfed children and their mothers, only one third of children under four months of age are breastfed exclusively. In addition, an overview of structures, actors, and measures to promote breastfeeding in Germany is missing. In a twoyear international research project entitled Becoming Breastfeeding Friendly (BBF), the current status of German breastfeeding support is systematically evaluated on the basis of the Breastfeeding Gear Model (BFGM) which was developed by the Yale School of Public Health. Therefore, committee members with expertise in the healthcare sector, science, policy, and communication evaluate 54 benchmarks of the eight relevant gears: advocacy, political will, legislation $\&$ policies, funding \& resources, training $\&$ program delivery, promotion, research \& evaluation, coordination, and goals \& monitoring. Based on the identified strengths and needs, concrete calls to action for scaling up breastfeeding promotion are derived and prioritized. BBF started in September 2017 and is conducted in cooperation with the Yale School of Public Health by the Healthy Start - Young Family Network as well as the National Breastfeeding Committee as an initiative of the Federal Ministry of Nutrition and Agriculture. The project assesses the framework conditions for breastfeeding and thus makes a valuable contribution to the health promotion of mother and child and to breastfeeding protection in Germany.

Keywords

Breastfeeding promotion - Becoming Breastfeeding Friendly $\cdot$ Healthy Start - Young Family Network - National Breastfeeding Committee 


\begin{tabular}{|c|c|c|}
\hline Handlungsfeld & Erläuterungen zum Handlungsfeld & $\begin{array}{l}\text { Anzahl der } \\
\text { Benchmarks }\end{array}$ \\
\hline \multirow[t]{2}{*}{ Anwaltschaft } & $\begin{array}{l}\text { Grad der Anwaltschaft (öffentliche Fürsprache durch Promi- } \\
\text { nente) zum Schutz und zur Förderung des Stillens }\end{array}$ & \multirow[t]{2}{*}{4} \\
\hline & $\begin{array}{l}\text { Beispiele: Veranstaltung großer Events mit medialer Auf- } \\
\text { merksamkeit auf Stillthemen; Einsatz für das Stillen durch } \\
\text { hochrangige Fürsprecher }\end{array}$ & \\
\hline \multirow[t]{2}{*}{ Politischer Wille } & $\begin{array}{l}\text { Verpflichtung/Engagement von staatlicher Seite zum Schutz } \\
\text { und zum Grad der Anwaltschaft (öffentliche Fürsprache } \\
\text { durch Prominente) zum Schutz und zur Förderung des Stil- } \\
\text { lens }\end{array}$ & \multirow[t]{2}{*}{3} \\
\hline & $\begin{array}{l}\text { Beispiele: öffentliche Unterstützung des Stillens durch po- } \\
\text { litische Entscheidungsträger; politische Initiativen zur Un- } \\
\text { terstützung einer stillfreundlichen gesellschaftlichen Atmo- } \\
\text { sphäre }\end{array}$ & \\
\hline \multirow[t]{2}{*}{ Gesetzgebung } & $\begin{array}{l}\text { Vorhandensein und Umsetzung von Gesetzen zum Schutz } \\
\text { und zur Förderung des Stillens }\end{array}$ & \multirow[t]{2}{*}{10} \\
\hline & $\begin{array}{l}\text { Beispiele: Integration der Initiative Babyfreundliche Kran- } \\
\text { kenhäuser in die Gesetzgebung; Gesetzgebung zum Mutter- } \\
\text { schutz, zu bezahlter Elternzeit und zur Einrichtung geeigne- } \\
\text { ter Stillräume am Arbeitsplatz; Umsetzung des Internationa- } \\
\text { len Kodex zur Vermarktung von Muttermilchersatzprodukten } \\
\text { in einem Land }\end{array}$ & \\
\hline \multirow[t]{2}{*}{ Finanzierung } & $\begin{array}{l}\text { Umfang und Angemessenheit von finanziellen und ander- } \\
\text { weitigen Ressourcen zum Schutz und zur Förderung des } \\
\text { Stillens }\end{array}$ & \multirow[t]{2}{*}{4} \\
\hline & $\begin{array}{l}\text { Beispiele: Vorhandensein eines nationalen Haushaltsbudgets } \\
\text { zur Förderung von Aktivitäten zum Schutz, zur Promotion } \\
\text { und Unterstützung des Stillens }\end{array}$ & \\
\hline \multirow[t]{2}{*}{ Bildung } & $\begin{array}{l}\text { Evaluation der Ausbildung und berufsbegleitenden Fort- und } \\
\text { Weiterbildung von Fachkräften im ambulanten und statio- } \\
\text { nären Sektor, die sich mit der Betreuung von Schwangeren, } \\
\text { jungen Müttern und Kleinkindern befassen }\end{array}$ & \multirow[t]{2}{*}{17} \\
\hline & $\begin{array}{l}\text { Beispiel: systematische Untersuchung der Lehrpläne von } \\
\text { stationären und ambulanten Einrichtungen zur Aus-, Fort- } \\
\text { und Weiterbildung sowie des Praxistrainings zu Stillthemen }\end{array}$ & \\
\hline \multirow[t]{2}{*}{ Werbung } & $\begin{array}{l}\text { Beurteilung der Qualität und Reichweite von Werbemaßnah- } \\
\text { men zum Schutz und zur Förderung des Stillens }\end{array}$ & \multirow[t]{2}{*}{3} \\
\hline & $\begin{array}{l}\text { Beispiele: mediale Kampagnen, öffentliche Veranstaltungen } \\
\text { und Auftritte in sozialen Netzwerken }\end{array}$ & \\
\hline \multirow{2}{*}{$\begin{array}{l}\text { Forschung \& } \\
\text { Evaluation }\end{array}$} & Bewertung von Monitoring- und Evaluierungssystemen & \multirow[t]{2}{*}{10} \\
\hline & $\begin{array}{l}\text { Beispiel: Vorhandensein eines standardisierten Stillmonito- } \\
\text { rings zur flächendeckenden Erfassung von Schlüsselindikato- } \\
\text { ren zum Stillverhalten }\end{array}$ & \\
\hline \multirow[t]{2}{*}{$\begin{array}{l}\text { Zielsetzung \& } \\
\text { Koordination }\end{array}$} & $\begin{array}{l}\text { Vorhandensein einer eigenständigen staatlichen Instituti- } \\
\text { on zur Koordination und Überprüfung aller Aktivitäten zur } \\
\text { Förderung des Stillens }\end{array}$ & \multirow[t]{2}{*}{3} \\
\hline & $\begin{array}{l}\text { Beispiele: Vorhandensein einer Nationalen Stillkommission; } \\
\text { Nutzung von Stillmonitoringdaten für Prozesse der Entschei- } \\
\text { dungsfindung; Prüfung des Aktionsplans der Nationalen } \\
\text { Stillkommission }\end{array}$ & \\
\hline
\end{tabular}

- Finanzierung,

- Bildung,

- Werbung,

- Forschung \& Evaluation,

- Zielsetzung \& Koordination.

Jedes der acht Zahnräder ist mit verschiedenen Prüfkriterien (sog. Benchmarks) zur Stillförderung hinterlegt. Insgesamt 54 Benchmarks werden untersucht. - Tab. 1 beschreibt die Handlungsfelder inhaltlich und gibt die Anzahl der Benchmarks pro Zahnrad an.

\section{Der BBF-Index (BBFI)}

Der BBFI ist eine nach einem standardisierten Verfahren erhobene Maßzahl, um den Stand der Stillförderung und Stillfreundlichkeit in einem Land auszudrücken. Er wurde durch die BBF-Steuerungsgruppe der Universität Yale (BBF Yale University Steering Committee) zusammen mit einer interdisziplinären wissenschaftlichen Arbeitsgruppe (BBF Technical Advisory Group - TAG) entwickelt [25]. Die Arbeitsgruppe (14 Mitglieder) besteht aus Wissenschaftlern (Bangladesch, Kanada, Ghana, Mexiko, Großbritannien und USA) sowie Führungskräften internationaler philanthropischer und gemeinnütziger Organisationen (Bill and Melinda Gates Foundation, Alive \& Thrive, The Manoff Group, GAIN und TAHN; [25]).

In einem ersten Schritt wurden potenzielle Themen und Benchmarks identifiziert, um jedes der acht Handlungsfelder erfassen zu können. Dazu wurde eine Übersicht über Schlüsselprojekte erstellt, die die Bereitschaft eines Landes hinsichtlich der Verstärkung von $\mathrm{Ge}$ sundheits- und Ernährungsinitiativen im Bereich Säuglings- und Kleinkindernährung, Lebensmittel und Ernährung sowie das Überleben Neugeborener untersuchen (PubMed und graue Literatur). Dabei wurden ausschließlich Schlüsselpublikationen und Initiativen berücksichtigt, die eine vollständige Beschreibung über die Methoden bzw. Mittel zur Verstärkung von Gesundheits- und Ernährungsinitiativen enthielten [25].

In einem Konsensprozess wurden die acht Handlungsfelder definiert, Themen identifiziert, die die Schlüsselkomponen- 


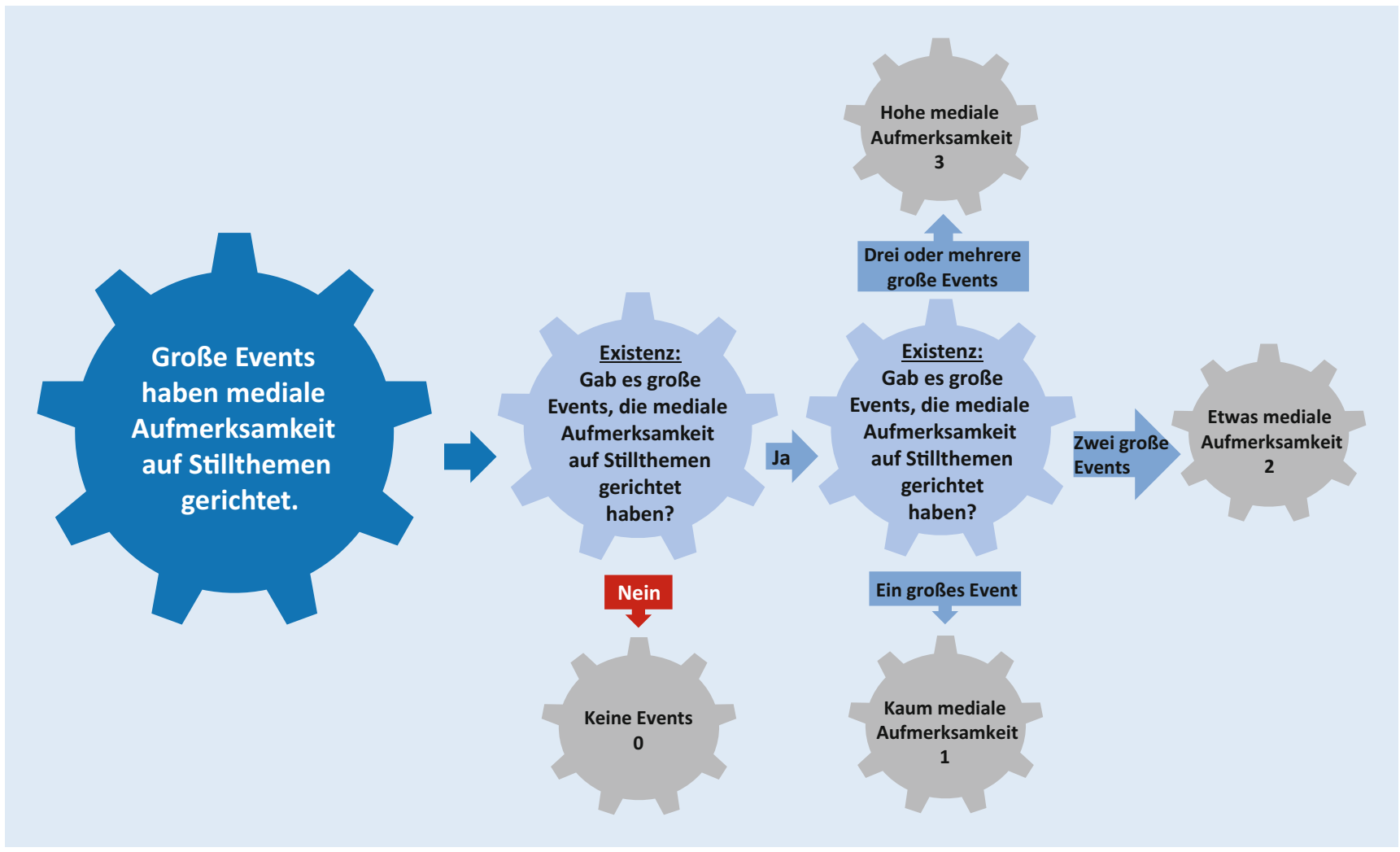

Abb. 2 ム Flowchart zur Bewertung eines ausgewählten Benchmarks des Handlungsfeldes „Anwaltschaft“ (mod. nach internen Dokumenten der Yale School of Public Health)

ten der Handlungsfelder widerspiegeln, sowie Benchmarks ermittelt, die diese Themen messen [25]. Der erste Entwurf des BBF-Index ergab eine Anzahl von insgesamt 129 Benchmarks.

Mithilfe der Delphi-Technik [26, 27] - einer Methode zur Entscheidungsfindung - wurde ein Konsens durch die Mitglieder der Technical Advisory Group über die Eignung der Benchmarks zur Erfassung von stillfreundlichen Rahmenbedingungen innerhalb eines Landes erzielt [25]. In einer finalen Version des BBFIndex wurden 54 Benchmarks beibehalten.

\section{Der BBFI-Score}

Das BBF-Scoring - ein System zur Bewertung der Handlungsfelder zur Stillförderung nach dem Breastfeeding Gear Model - ist die wissenschaftliche Grundlage für die Stärken- und Schwächenanalyse der jeweiligen nationalen Rahmenbedingungen zur Stillförderung [25].

\section{Schritt 1: Die Bewertung der Benchmarks}

In einem ersten Schritt werden alle 54 Benchmarks nach bestimmten Kriterien auf einer Skala von 0 bis 3 Punkten bewertet. Ein Punktewert von 0 beschreibt dabei das Nichterfüllen des jeweiligen Kriteriums, ein Wert von 3 signalisiert, dass das Kriterium erfüllt ist (• Abb. 2). Die Bewertung bezieht sich rückblickend auf ein Jahr (in der Regel das vergangene Jahr).

Beispiel: Im ersten Benchmark des Handlungsfeldes „Anwaltschaft“ wird geprüft, ob große Events mediale Aufmerksamkeit auf Stillthemen gerichtet haben. Nach folgenden Kriterien wird bewertet:

1. Kein großes Event hat national mediale Aufmerksamkeit auf Stillthemen gerichtet.

2. Es gab ein großes Event, das national mediale Aufmerksamkeit auf Stillthemen gerichtet hat.

3. Es gab zwei große Events zu verschiedenen Zeitpunkten innerhalb eines Jahres, die national mediale
Aufmerksamkeit auf Stillthemen gerichtet haben.

4. Es gab drei oder mehr große Events zu verschiedenen Zeitpunkten innerhalb eines Jahres, die national mediale Aufmerksamkeit auf Stillthemen gerichtet haben.

\section{Schritt 2: Die Bewertung der acht Handlungsfelder (GTS, Gear Total Score)}

Nach dem Scoring jedes der 54 Benchmarks wird für jedes Zahnrad ein spezifischer GTS berechnet, der auch alleinstehend interpretiert werden kann. Er beschreibt die Stillfreundlichkeit für jedes der acht Zahnräder. Bei der Berechnung des GTS wird die Anzahl der Benchmarks pro Zahnrad berücksichtigt.

Beispiel: GTS politischer Wille= Summe aller Benchmarkscores für dieses Zahnrad/3 (= Anzahl der Benchmarks dieses Zahnrads).

Der Gear Total Score wird wie folgt interpretiert: 


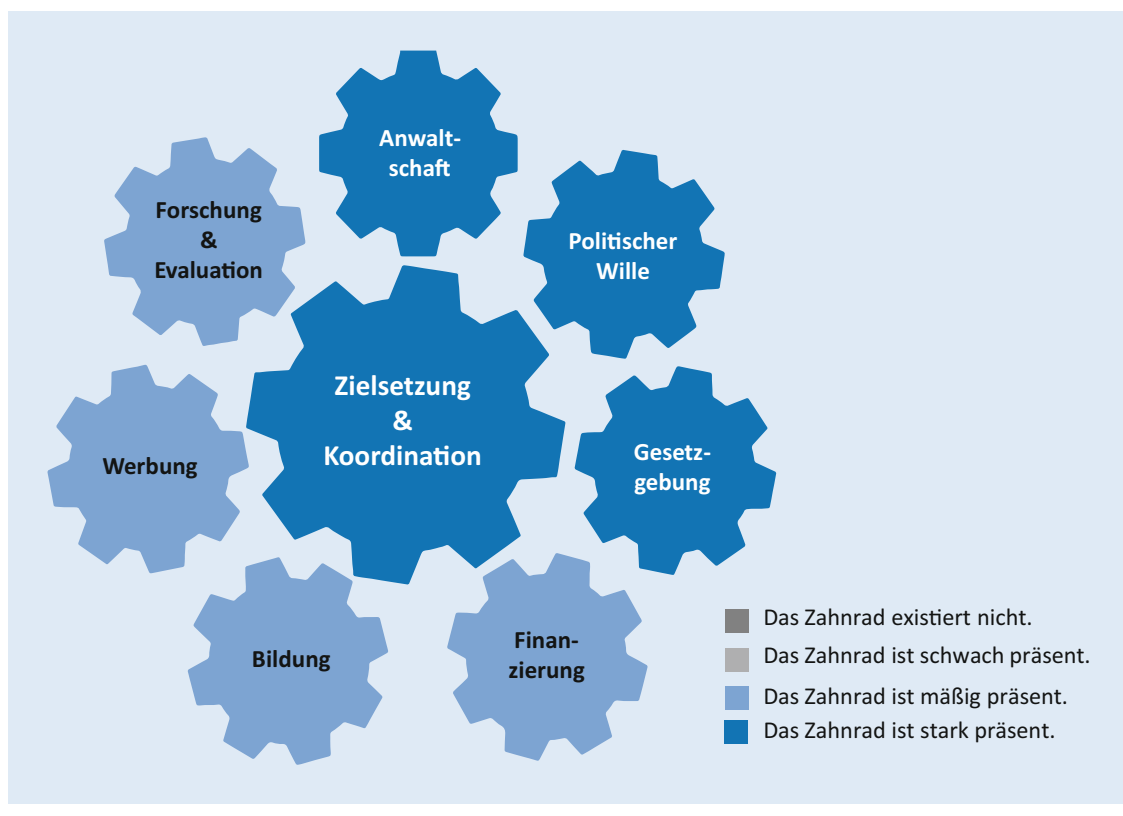

Abb. $3 \Delta$ Bewertung der Handlungsfelder zur Stillförderung nach dem Breastfeeding Gear Model am Beispiel Ghana (mod. nach [28])

- 0: Das Zahnrad existiert nicht.

- 0,1 bis 1,0: Das Zahnrad ist schwach präsent.

- 1,1 bis 2,0: Das Zahnrad ist mäßig präsent.

- 2,1 bis 3,0: Das Zahnrad ist stark präsent.

Der GTS deckt die Stärken und Schwächen der einzelnen Handlungsfelder innerhalb eines Landes auf und gibt gezielte Hinweise, in welchen Bereichen in Stillförderung investiert werden sollte.

\section{Schritt 3: Berechnung des BBFI Total Score (BBFI-TS)}

Auf Basis aller GTS wird der finale BBFI-TS eines Landes berechnet. Hierbei findet eine Gewichtung einzelner Zahnräder anhand durchschnittlicher Rankingscores zum Einfluss der Benchmarks (1,4: niedrig, 1,5: mittel, 1,6: hoch) Anwendung. Zur Bestimmung der Gewichtung haben die Mitglieder der Technical Advisory Group alle 54 Benchmarks hinsichtlich ihres Einflusses ( $1=$ niedrig, $2=$ mittel, $3=$ hoch) auf die Stillfreundlichkeit bewertet [25]. Anhand der durchschnittlichen Rankings der Benchmarks wird folglich der Einfluss jedes einzelnen Benchmarks ersichtlich. In einem weiteren Schritt wurde die Gewichtung der Zahnrä-
- 1,1 bis 2,0: mäßig stillförderndes Umfeld,

- 2,1 bis 2,9: sehr stillförderndes Umfeld,

- 3,0: herausragend stillförderndes Umfeld.

Dieses standardisierte Verfahren ermöglicht eine Verlaufsbeobachtung der Aktivitäten zur Stillförderung in einem Land sowie einen Vergleich und Erfahrungsaustausch zu Aktivitäten der Stillförderung in anderen Ländern. Das Projekt wurde bereits in Mexiko und Ghana durchgeführt. Zeitgleich mit Deutschland haben Myanmar, Samoa, Großbritannien (England, Schottland und Wales) begonnen.

In Ghana ist das Forschungsvorhaben im Mai 2016 gestartet. Die Ergebnisse wurden auf einem Kongress im Februar 2017 vorgestellt [28]. • Abb. 3 zeigt die Bewertung der einzelnen Handlungsfelder (Gear Total Score) für Ghana.

die Benchmark hinsichtlich ihres Zahnrades gruppiert wurden und deren Durchschnittswert berechnet wurde.

Jeder GTS wird wie folgt gewichtet:

- gewichteter GTS Anwaltschaft = GTS

Anwaltschaft $\times 1,6$,

- gewichteter GTS politischer Wille $=$ GTS politischer Wille $\times 1,5$,

- gewichteter GTS Gesetzgebung= GTS Gesetzgebung $\times 1,6$,

- gewichteter GTS Finanzierung= GTS Finanzierung $\times 1,6$,

- gewichteter GTS Bildung=GTS Bildung $\times 1,6$,

- gewichteter GTS Werbung=GTS Werbung $\times 1,5$,

- gewichteter GTS Forschung \& Evaluation $=$ GTS Forschung \& Evaluation $\times 1,5$,

- gewichteter GTS Zielsetzung \& Koordination $=$ GTS Zielsetzung \& Koordination $\times 1,4$.

Abschließend werden alle acht gewichteten GTS addiert und durch die Summe aller Zahnradgewichtungen $(=12,3)$ dividiert. Der resultierende BBFI-TS drückt die Stärke der Stillfreundlichkeit eines Landes aus und wird wie folgt interpretiert:

- 0 bis 1,0: schwach stillförderndes Umfeld,

\section{Ablauf des Forschungs- vorhabens}

\section{Initial-/Vorbereitungsphase}

Das Vorgehen von Becoming Breastfeeding Friendly in Deutschland wurde vom Netzwerk Gesund ins Leben und der Nationalen Stillkommission in Kooperation mit der Yale School of Public Health abgestimmt und an die nationalen Verhältnisse angepasst. Zu Beginn des Vorhabens wurde vom Projektteam eine erste Datenrecherche zu Informationen über die acht Handlungsfelder durchgeführt. Eine gezielte Öffentlichkeitsarbeit wurde genutzt, um auf das Forschungsvorhaben sowie die Notwendigkeit der Stillförderung in Deutschland aufmerksam zu machen.

Darüber hinaus wurde eine Kommission aus nationalen Experten gebildet, die eine ausgewiesene Expertise und/oder eine Stakeholder-Funktion für bestimmte Handlungsfelder aufweisen. Die Kommission setzt sich zusammen aus Vertretern von

- Fachgesellschaften der mit jungen

Müttern und Säuglingen befassten

Gesundheitsberufe (Hebammen, Gy- 


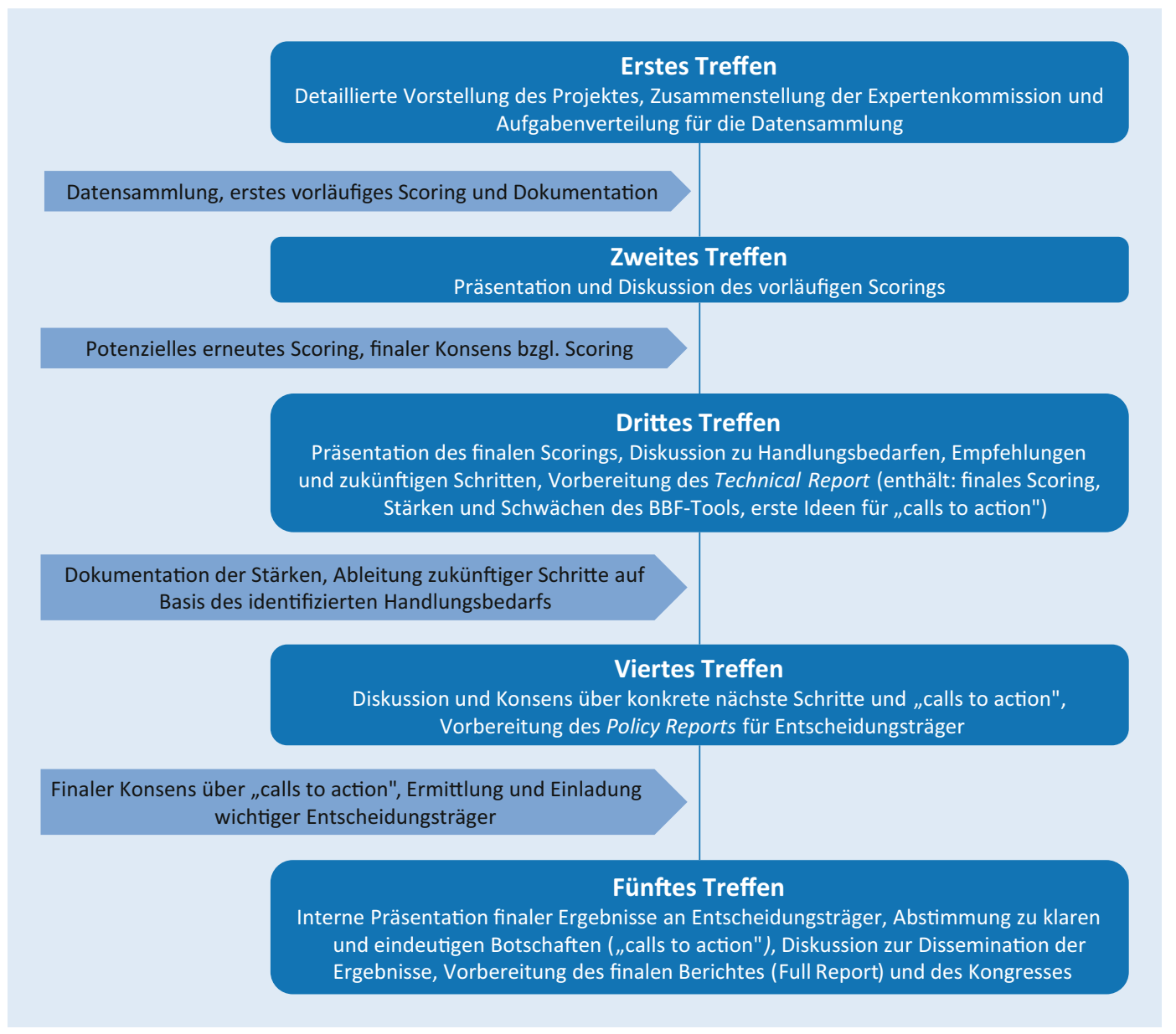

Abb. $4<$ Arbeitstreffen zur Datenerhebung, Bewertung und zum Scoring der Informationen näkologen, Kinder- und Jugendärzte, Pflege),

- Bundesministerien (Ernährung, Familie, Gesundheit),

- Kommunen, Öffentlichem Gesundheitsdienst, Frühen Hilfen,

- Medien, Gesundheitskommunikation, Stillmonitoring.

Die Besetzung der Expertenkommission kann unter folgendem Link eingesehen werden: https://www.gesund-ins-leben. de/inhalt/experten-kommission-31019. html.

\section{Datenerhebung- und Aus- wertungsphase/Expertenprozess}

Die Datenerhebung und -auswertung der Informationen zu den acht Handlungsfeldern erfolgt im Rahmen eines mehrstufigen Prozesses. Dieser startete im März 2018 und verläuft nach einem von der Yale School of Public Health erprobten Schema von etwa fünf ein- bis zweitä- gigen Arbeitstreffen (• Abb. 4). Zu Beginn des Prozesses bestimmt die Expertenkommission die für das Scoring notwendigen Datenquellen und recherchiert diese. Anschließend bewerten die Experten alle 54 Benchmarks nach definierten Kriterien und belegen in einem Konsens abschließend jedes Zahnrad mit einem finalen GTS. Das Scoring sowie die identifizierten Schwachstellen (Gaps) sind dann Grundlage, um am Ende des Prozesses Handlungsempfehlungen für die verschiedenen Zahnräder zu erstellen. Nach bestimmten Kriterien der Forschungsinitiative für Kindergesundheit und Ernährung (CHNRI; [29]) werden die wichtigsten Empfehlungen priorisiert und klare und eindeutige Botschaften („calls to action“) erarbeitet. Hierbei stellt die Expertenkommission im dritten Treffen eine Liste aller Handlungsempfehlungen zur Diskussion vor. Die Liste dieser Empfehlungen bildet die Grundlage für das Priorisierungsverfahren. Dieses erfolgt aufder Basis der drei Kriterien Wirk- samkeit, Finanzierbarkeit und Machbarkeit zunächst in einem standardisierten Onlinebewertungsverfahren durch einzelne Mitglieder der Kommission. Die Ergebnisse der Einstufung werden dann im vierten Treffen allen Mitgliedern präsentiert. Ziel ist es, am Ende des vierten Treffens 3-5 prioritäre Handlungsempfehlungen im Konsens festzulegen.

\section{Umsetzungsphase: Verwertung der Ergebnisse}

Die Erkenntnisse der Analyse über Stärken und Handlungsbedarfe werden genutzt, um darauf aufbauend für Deutschland maßgeschneiderte Stillfördermaßnahmen abzuleiten, zu initiieren und $\mathrm{zu}$ begleiten. Auf Basis der von der Expertenkommission entwickelten Handlungsempfehlungen („calls to action") soll daher ein breiter gesellschaftlicher Dialog und Aufruf folgen, um die Stillförderung in allen Handlungsfeldern zu optimieren. Hierbei ist ein Mitwirken 
vieler Akteure im Bereich der Mütterund Kindergesundheit erforderlich, aber auch die Unterstützung von politischer Seite unabdingbar.

Geplant ist eine Tagung mit relevanten Akteuren: Entscheidungsträgern und Experten zu den acht Handlungsfeldern, Vertretern aus Politik und Medien sowie föderalen Ebenen (Bund, Länder und Kommunen). Ziel der Veranstaltung ist, die Scoringergebnisse und die abgeleiteten Handlungsempfehlungen vorzustellen. In einem partizipativen Prozess werden die Ergebnisse mit den Teilnehmern der Konferenz diskutiert und priorisiert. In einem weiteren Schritt sollen gemeinsam mit den Akteuren Ideen und Maßnahmen entwickelt werden, mit denen diese in ihren jeweiligen Handlungsfeldern dazu beitragen können, ein stillfreundlicheres Klima in Deutschland zu schaffen. Dies erfolgt, indem über die verschiedenen Handlungsfelder (Zahnräder) und die föderalen Strukturen möglichst breit und umfassend die Schnittstellen zur Stillförderung erreicht werden.

\section{Begleitung und Monitoring der Maßnahmen}

Aus Gründen der Transparenz, Effizienz und Nachhaltigkeit werden die im Rahmen dieses Vorhabens angestoßenen Maßnahmen begleitet und koordiniert. Sowohl das Netzwerk Gesund ins Leben als auch die Nationale Stillkommission unterstützen die Organisationen und Institutionen bedarfsorientiert bei der Maßnahmendurchführung durch Beratung, Begleitung und Dokumentation. Auch Evaluationsaktivitäten werden auf diese Weise bereits während der Vorhabenlaufzeit angestoßen.

\section{Öffentlichkeitsarbeit und Dissemination}

Unter dem Motto: „Tue Gutes und rede darüber", erfolgt eine breit angelegte Kommunikationsarbeit. Diese richtet sich an die Bevölkerung, an Fachkreise, Politik und Medien, um die Wahrnehmung des Stillens und seiner gesundheitlichen Vorteile zu erhöhen sowie die Ergebnisse des BBF-Vorhabens bekannt zu machen. Auch soll damit ein gesell- schaftliches Klima geschaffen werden, in dem stillende Frauen die notwendige Unterstützung und Wertschätzung erhalten. Das Disseminationskonzept beinhaltet u.a. das Verfassen von Fachartikeln, Internetinhalten und Pressemitteilungen sowie die Präsenz und Vorträge/Workshops auf Tagungen und Kongressen. Genutzt werden die Verbreitungskanäle des Netzwerks Gesund ins Leben und der Nationalen Stillkommission sowie möglichst vieler weiterer Partner des BBF-Vorhabens.

\section{Das internationale Breast- feeding-Gear-Modell in Deutschland}

Das Projekt Becoming Breastfeeding Friendly bietet eine große Chance, nach international einheitlichen Kriterien Faktoren der Stillförderung in Deutschland zu evaluieren, um folglich gezielte Handlungsbedarfe zu identifizieren und konkrete Handlungsempfehlungen („,calls to action") zu formulieren. Durch die kritische Auseinandersetzung mit den insgesamt 54 Benchmarks des BFGM wird sichtbar, wo gezielte Maßnahmen ansetzen sollten. Die von der Universität Yale bereitgestellte Toolbox ermöglicht jedem Land, das Projekt nach gleichen Kriterien durchzuführen. Eine internationale Vergleichbarkeit der Ergebnisse wird jedoch durch unterschiedliche länderspezifische Gegebenheiten, wie beispielsweise das Gesundheitssystem, limitiert. Gleichzeitig ist die Expertenkommission dazu angehalten, Anpassungen an länderspezifische Gegebenheiten vorzunehmen und zu dokumentieren. Deutschland ist eine der ersten Industrienationen, in der das Projekt durchgeführt wird. Die kritische und transparente Auseinandersetzung mit dem Modell kann die Umsetzung des Projektes für nachfolgende Länder erleichtern.

Die Besetzung der Expertenkommission hat einen entscheidenden Einfluss auf die Bewertung der Faktoren der Stillförderung und somit auf die Entwicklung von Handlungsempfehlungen. Bei der Besetzung der deutschen Kommission wurde großer Wert auf eine ausgeglichene Beteiligung von Experten aus den verschiedenen Handlungsfeldern der Stillförderung gelegt. Gleichzeitig erleichtert die Einbeziehung solcher Stakeholder die spätere Umsetzung von Maßnahmen der Stillförderung.

\section{Ausblick}

Trotz des hinreichend belegten gesundheitsförderlichen Potenzials ist das Stillen von Säuglingen in Deutschland nicht die Regel. Es benötigt umfangreiche und ressourcenorientierte Maßnahmen, um das Stillen zu schützen, zu unterstützen und zu bewerben - angefangen mit einer stillfreundlichen Gesetzgebung und Politik über die verstärkte Unterstützung von berufstätigen Müttern und deren Familien bis hin zu einer Verbesserung der Aus-, Weiter- und Fortbildung von Fachpersonal zum Stillen.

Mithilfe des Forschungsvorhabens Becoming Breastfeeding Friendly wird die aktuelle Situation der Stillförderung in Deutschland für das Jahr 2017 abgebildet: Welche Initiativen und Maßnahmen der Öffentlichkeitsarbeit gibt es bereits? Wie berichten Massenmedien über das Thema Stillen und Muttermilch? Welche Strukturen werden für die Stillförderung genutzt? Welche Akteure geben wichtige Ideen und Impulse? Wie umfangreich wird das Fachpersonal in den Gesundheits- und Sozialberufen zu stillbezogenen Lerninhalten aus- und weitergebildet und sind diese Inhalte in den Lehrplänen verankert? Als Datengrundlage für das Scoring der Benchmarks werden daher u. a. eine quantitative Inhaltsanalyse der Medienberichterstattung in Print, TV und Social Media durchgeführt, zudem findet eine systematische Befragung von Aus-, Fort- und Weiterbildungsinstitutionen für Gesundheitsfachberufe sowie Sozialberufe statt, die mit der Unterstützung von Schwangeren und Müttern von Säuglingen betraut sind. Die Erkenntnisse aus den Scorings liefern fundierte Daten für die künftige Planung, Initiierung und Begleitung von Maßnahmen und Kampagnen für die Stillförderung und den Stillschutz.

Derzeit ist es nicht möglich, die Entwicklung des Stillverhaltens in Deutschland zeitnah und zuverlässig einzuschätzen. Stillhäufigkeiten und -dauer werden überwiegend lokal oder regional 
sowie mit verschiedenen Zielsetzungen und Methoden erhoben [30]. Das Forschungsvorhaben Becoming Breastfeeding Friendly könnte die Etablierung eines standardisierten Stillmonitorings in Deutschland unterstützen, welches repräsentativ und kontinuierlich das Stillverhalten abbildet und die Daten in Beziehung zu den nationalen Aktivitäten der Stillförderung setzt. Ein standardisiertes Stillmonitoring soll zukünftig mit zum Aufgabenbereich des neu gegründeten Instituts für Kinderernährung gehören. Das Institut für Kinderernährung wird am Hauptstandort des Max Rubner-Instituts in Karlsruhe angesiedelt.

Die Initiatoren von Becoming Breastfeeding Friendly bezeichnen schon die Durchführung des Vorhabens selbst als eine Art der Intervention - inklusive Auswahl der Experten, Erstellung der Kommission, Sammlung aller relevanten Daten, Einigung auf das Scoring, Ableitung von Empfehlungen und „calls to action“. Allein die intensive und interdisziplinäre Auseinandersetzung mit dem Thema auf unterschiedlichen Ebenen und der Prozess der Konsensfindung führen zu einer verstärkten Vernetzung verschiedenster Akteure.

Die potenziellen Auswirkungen der durch das Vorhaben angestoßenen Maßnahmen und Initiativen können erst über einen längerfristigen Beobachtungszeitraum hinweg ermittelt werden. Maßnahmen, die auf der Grundlage von BBF initiiert wurden, sollten zentral begleitet und monitoriert werden. Im Rahmen eines Follow-ups des BBF-Vorhabens könnten die getroffenen Maßnahmen nach einem bestimmten Zeitraum evaluiert und eventueller Nachsteuerungsbedarf bzgl. der Stillförderung in Deutschland ermittelt werden.

Durch die partizipative Arbeitsweise und das systematische Einbinden und Vernetzen relevanter Akteure von Beginn des Prozesses an können tragende Strukturen für die Stillförderung geschaffen und langfristig etabliert werden. Denkbar ist zudem, dass aus den recherchierten und den im Rahmen des Vorhabens initiierten Maßnahmen zur Stillförderung eine Art Datenbank erstellt wird, die so- wohl national als auch international zugänglich gemacht wird.

Damit kann dieses Forschungsvorhaben einen wertvollen Beitrag zur Gesundheitsförderung von Mutter und Kind leisten und zum Stillschutz in Deutschland beitragen.

Nähere Informationen zum Vorhaben erhalten Sie hier:

$$
\begin{aligned}
& \text { - www.gesund-ins-leben.de/ } \\
& \text { becoming-breastfeeding-friendly } \\
& \text { - http://www.bbf.yale.edu/ }
\end{aligned}
$$

\section{Korrespondenzadresse}

\section{Flothkötter}

Netzwerk Gesund ins Leben/Bundeszentrum für Ernährung (BZfE) in der Bundesanstalt für Landwirtschaft und Ernährung (BLE) Deichmanns Aue 29, 53179 Bonn, Deutschland maria.flothkoetter@ble.de

Förderung. Das Forschungsvorhaben wird mit Mitteln des Bundesministeriums für Ernährung und Landwirtschaft finanziert. Die Yale School of Public Health begleitet den gesamten Prozess wissenschaftlich. Für diese Zusammenarbeit erhält das Netzwerk Gesund ins Leben von der Yale School of Public Health eine einmalige Förderung aus Mitteln der Familie Larsson-Rosenquist Stiftung.

\section{Einhaltung ethischer Richtlinien}

Interessenkonflikt. M. Flothkötter, J. Kunath, S. Lücke, K. Reiss, J. Menzel und C. Weikert geben an, dass kein Interessenkonflikt besteht.

Dieser Beitrag beinhaltet keine von den Autoren durchgeführten Studien an Menschen oder Tieren.

Open Access. Dieser Artikel wird unter der Creative Commons Namensnennung 4.0 International Lizenz (http://creativecommons.org/licenses/by/4.0/deed. de) veröffentlicht, welche die Nutzung, Vervielfältigung, Bearbeitung, Verbreitung und Wiedergabe in jeglichem Medium und Format erlaubt, sofern Sie den/die ursprünglichen Autor(en) und die Quelle ordnungsgemäßnennen, einen Linkzur Creative Commons Lizenz beifügen und angeben, ob Änderungen vorgenommen wurden.

\section{Literatur}

1. Koletzko B, Bauer CP, Cierpka M et al (2016) Ernährung und Bewegung von Säuglingen und stillenden Frauen. Aktualisierte Handlungsempfehlungen von "Gesund ins Leben - Netzwerk Junge Familie", eine Initiative von IN FORM. Monatsschr Kinderheilkd 164(9):765-789

2. Chowdhury R, Sinha B, Sankar MJ et al (2015) Breastfeeding and maternal health outcomes: a systematic reviewand meta-analysis. ActaPaediatr 104:96-113

3. Dieterich CM, Felice JP, O'Sullivan E et al (2013) Breastfeeding and health outcomes for the mother-infant dyad. Pediatr Clin North Am 60:31-48

4. Horta BL, Victora CG (2013) Long-term effects of breastfeeding. A systematic review. http://apps.who.int/iris/bitstream/10665/79198/ 1/9789241505307_eng.pdf.Zugegriffen: 18. Jan. 2018

5. Horta BL, Loret de Mola C, Victora CG (2015) Long-term consequences of breastfeeding on cholesterol, obesity, systolic blood pressure and type 2 diabetes: a systematic review and metaanalysis. Acta Paediatr Suppl 104:30-37

6. Ip S, Chung M, Raman G et al (2009) A summary of the Agency for Healthcare Research and Quality's evidence report on breastfeeding in developed countries. Breastfeed Med 4(Suppl 1):S17-S30

7. Victora CG, Bahl R, Barros AJD et al (2016) Breastfeeding in the 21th century: epidemiology, mechanisms, and lifelong effect. Lancet 387:475-490

8. Rollins NC, Bhandari N, Hajeebhoy N et al (2016) Why invest, and what it will take to improve breastfeeding practices? Lancet 387:491-504

9. Brettschneider AK, Weikert C, Abraham K et al (2016) Stillmonitoring in Deutschland - Welchen Beitrag können die KiGGS-Daten leisten? J Health Monit 1(2):16-25

10. von der Lippe $E$, Brettschneider AK, Gutsche J et al (2014) Einflussfaktoren auf Verbreitung und Dauer des Stillens in Deutschland -Ergebnisse der KiGGSStudie - Erste Folgebefragung (KiGGS Welle 1). Bundesgesundheitsblatt Gesundheitsforschung Gesundheitsschutz 57(7):849-859

11. Lange C, Schenk L, Bergmann R (2007) Verbreitung, Dauer und zeitlicher Trend des Stillens in Deutschland. Bundesgesundheitsblatt Gesundheitsforschung Gesundheitsschutz 50:624-633

12. Unicef (1990) Innocenti declaration-on the protection, promotion and support of breastfeeding. https://www.unicef.org/programme/ breastfeeding/innocenti.htm. Zugegriffen: 26. Febr. 2018

13. World Health Organization, UNICEF (2009) BabyFriendly Hospital Initiative-revised, updated and expanded for integrated care. http://www.who. int/nutrition/publications/infantfeeding/bfhi_ trainingcourse/en/.Zugegriffen:26. Febr. 2018

14. World Health Organization (2014) Global nutrition targets 2025: breastfeeding policy brief. http:// www.who.int/iris/bitstream/10665/149022/1/ WHO_NMH_NHD_14.7_eng.pdf.Zugegriffen: 24 . Jan. 2018

15. World Health Organization (2013) Regional Office for Europe. Stillende Mütter benötigen Beratung aus ihrem persönlichen Umfeld. http://www.euro. who.int/en/health-topics/Life-stages/pages/ news/news/2013/08/breastfeeding-mothersneed-peer-counselling. Zugegriffen: 17. Jan. 2018

16. World Health Organization (2015) Regional Office for Europe. Europäische Region der WHO hat niedrigste Stillraten weltweit. http://www.euro.who.int/de/health-topics/ noncommunicable-diseases/obesity/news/ news/2015/08/who-european-region-haslowest-global-breastfeeding-rates. Zugegriffen: 17. Jan. 2018

17. Bundesinstitut für Risikobewertung (Hrsg) (2017) Positive Botschaften für ein ungestörtes Stillen in der Öffentlichkeit. Stellungnahme der Nationalen Stillkommission. http://www.bfr. 
bund.de/cm/343/positive-botschaften-fuer-einungestoertes-stillen-in-der-oeffentlichkeit.pdf (Erstellt:29. Juni 2017).Zugegriffen:7. Febr. 2018

18. Rückert IM, Mielck A (2008) Soziale Ungleichheit beim Stillen in Deutschland. Präv Gesundheitsf 3(2):56-66

19. World Health Organization, United Nations Children's Fund, Global Breastfeeding Collective (2017) Global breastfeeding scorecard, 2017: tracking progress for breastfeeding policies and programmes. www.who.int/nutrition/.../globalbf-scorecard-2017.pdf. Zugegriffen: 25. Mai 2018

20. World Breastfeeding Trends Initiative (2018) The guide book: world breastfeeding trends initiative (WBti) - galvanising action on breastfeeding and Infant and Young Children Feeding (IYCF). http://worldbreastfeedingtrends.org/guidebook-wbti/.Zugegriffen: 25. Mai 2018

21. IBFAN (2014) World Breastfeeding Trends Initiative (WBTi) assessment tool: IBFAN (Asia)

22. WABA (1993) The global participatory action research (GLOPAR) project. World Alliance for Breastfeeding Action

23. Pérez-Escamilla R, Hromi-Fiedler AJ, Gubert MB et al (2018) Becoming Breastfeeding Friendly Index: development and application for scaling-up breastfeeding programmes globally. Matern Child Nutr 14:e12596

24. Pérez-Escamilla R, Hall Moran V (2016) Scaling up breastfeeding programmes in a complex adaptive world. Matern Child Nutr 12:375-380

25. Pérez-Escamilla R, Curry L, Minhas DT et al (2012) Scaling up of breastfeeding promotion programs in low- and middle-income countries: the "breastfeeding gear" model. Adv Nutr 3:790-800

26. Chian-Chen Hsu BAS (2007) The Delphi technique: making sense of consensus. Pract Assess Res Eval 12:10

27. OkoliC, PawlowskiSD (2004) The Delphimethodas a research tool: an example, design considerations and applications. InfManage 42(1):15-29

28. Ghana BBF (2017) Ghana BBF policy brief. Becoming breastfeeding friendly-recommended actions to scale up breastfeeding impact in Ghana. http://medicine.yale.edu/ysph/bfci/countries/ Ghana\%20policy\%20brief\%20KD\%20edits\%208. 25.17_314242_284_34128_v1.pdf.Zugegriffen: 8. Febr. 2018

29. Rudan I, Chopra M, Kapiriri L et al (2008) Setting priorities in global child health research investments: universal challenges and conceptual framework. Croat Med J 49:307-317

30. Bundesinstitut für Risikobewertung (2017) Nationale Stillkommission diskutiert Wege zu einem standardisierten Stillmonitoring für Deutschland. http://www.bfr.bund.de/cm/343/ nationale-stillkommission-diskutiert-wege-zueinem-standardisierten-stillmonitoring-fuerdeutschland.pdf.Zugegriffen:24. Mai 2018 